\title{
The relationship between neurocysticercosis and epilepsy: an endless debate
}

\author{
A relação entre neurocisticercose e epilepsia: um debate interminável \\ Arturo Carpio ${ }^{1,2}$, Matthew L. Romo ${ }^{3}$
}

\begin{abstract}
Neurocysticercosis (NC), or cerebral infection with Taenia solium, is an important public health problem worldwide. Among the neurological sequelae of $\mathrm{NC}$, seizures have been described as the most common symptom. Acute symptomatic seizures often result from degeneration of a viable cyst; however, not all of these patients with acute or provoked seizures will develop epilepsy (i.e., recurrent unprovoked seizures). Because of the high prevalence of epilepsy and NC, a causal, as well as incidental relationship between the two may exist. The epileptogenicity of calcified cysts as well as the potential association between NC and hippocampal sclerosis necessitates future research. Antihelminthic treatment of NC results in disappearance of viable cysts in about one-third of patients with parenchymal disease, but a reduction in seizure recurrence has not been demonstrated in randomized controlled trials. Prevention is critical to reduce the burden of seizure and epilepsy related to NC.
\end{abstract}

Keywords: cysticercosis, Taenia solium, seizure, parasitic.

\section{RESUMO}

A neurocisticercose (NC) ou infecção cerebral pela Taenia solium é importante problema de saúde pública em todo o mundo. Entre as sequelas neurológicas da NC, as crises convulsivas têm sido descritas como os sintomas mais frequentes. Crises convulsivas sintomáticas agudas resultam, muitas vezes, da degeneração de cistos viáveis; entretanto, nem todos os pacientes com NC e crises agudas ou provocadas desenvolvem epilepsia (i.e., crises recorrentes não provocadas). Pode haver uma relação entre epilepsia e NC, causal ou incidental, devido à alta prevalência de ambas. 0 potencial epileptogênico dos cistos calcificados assim como a possível associação entre NC e esclerose hipocampal ainda necessitam de futuras pesquisas. O tratamento anti-helmíntico da NC resulta no desaparecimento de cistos viáveis em cerca de 1/3 dos pacientes com a doença parenquimatosa, mas não foi demonstrada redução nas taxas de recorrência das crises convulsivas em estudos randomizados controlados. A prevenção é crítica para reduzir o contingente de crises convulsivas e epilepsia relacionadas à NC.

Palavras-chave: cisticercose, Taenis solium, convulsão, parasítico.

Neurocysticercosis (NC) is a parasitic infection of the central nervous system (CNS) with Taenia solium and can be viewed as a biological marker of poverty and underdevelopment ${ }^{1}$. Transmission of $T$. solium has been traditionally reported in endemic regions of Latin America, Asia and Africa $^{2}$; however, it is becoming an emerging infectious disease in non-endemic, high-income or developed countries, primarily due to immigration from endemic regions ${ }^{3}$. Infection with $T$. solium larvae occurs through consumption of the eggs shed by humans who have become carriers of $T$. solium, either by person-to-person transmission or auto-infection. In addition to NC, epilepsy is also common in developing countries or low-income countries, but the two pathologies are not always related. The global prevalence of NC is not well understood, but it is often asserted that NC is the leading cause of epilepsy in endemic regions. It is well known that $\mathrm{NC}$ and epilepsy independently have a significant impact on morbidity and mortality, but are also a source of high economic burden, particularly for low-income or developing countries $^{2}$. In the context of $\mathrm{NC}$, it is critical to differentiate between seizures and epilepsy. Acute symptomatic seizures are the most common clinical manifestation of symptomatic $\mathrm{NC}$; however, the causal link between NC and epilepsy needs to be carefully analyzed. In this critical review, we will evaluate

${ }^{1}$ School of Medicine, University of Cuenca, Cuenca, Ecuador;

${ }^{2} \mathrm{GH}$ Sergievsky Center, College of Physicians and Surgeons, Columbia University, New York, USA;

${ }^{3}$ CUNY School of Public Health, New York, USA.

Correspondence: Arturo Carpio; Consultorios Santa Inés, Córdova y F. Proaño (esquina) (4to piso); Cuenca, Ecuador; E-mail: arturo.carpio@ucuenca.edu.ec Conflict of interest: There is no conflict of interest to declare.

Received 27 January 2014; Accepted 17 February 2014. 
the evidence for the epileptogenicity of NC and identify the gaps in the current scientific literature.

\section{DISTINGUISHING BETWEEN SEIZURES AND EPILEPSY AS THEY RELATE TO NEUROCYSTICERCOSIS}

Establishing the essential features, distinctive characteristics and uniform definitions of seizures, epilepsy and NC, are crucial to facilitate accurate diagnosis and effective treatment and prevention. According to the definitions suggested by the International League Against Epilepsy (ILAE), epilepsy is defined as "two or more unprovoked seizures occurring at least 24 hours apart"4. Unprovoked seizures may occur subsequently to a well-demonstrated antecedent condition, which is known to substantially increase the risk of epileptic seizures ${ }^{4}$. The ILAE further divide the causes of epilepsy into three broad categories: genetic, structural/metabolic, and unknown ${ }^{5}$. The structural/metabolic category includes when a structural lesion (either static or progressive) or metabolic condition (e.g., inborn errors of metabolism) is present and is known to be associated with an increased risk of epilepsy ${ }^{5}$. In other words, the cause of epilepsy is attributed to the condition that is most directly linked to the development of epilepsy. In the context of $\mathrm{NC}$, this might include patients with epilepsy that have calcified parenchymal cysts present on imaging.

An epileptic seizure is defined as "a transient occurrence of signs and/or symptoms due to abnormal excessive or synchronous neuronal activity in the brain"6. Epileptic seizures, even if recurrent, are not always synonymous with epilepsy ${ }^{4}$. Provoked or acute symptomatic seizures occur "in close temporal association with an acute systemic, metabolic, or toxic insult or in association with an acute CNS insult (infection, stroke, cranial trauma, intracerebral hemorrhage, or acute alcohol intoxication or withdrawal)" ${ }^{6,7}$. Such seizures are often isolated events associated with acute conditions, but may recur if the acute condition recurs. Seizures associated with NC may be categorized as either acute symptomatic seizures or as unprovoked symptomatic seizures. Acute symptomatic seizures may result from transitional or degenerating cysts, but are ultimately a consequence of the acute inflammatory response. In contrast, a patient with seizures who has viable cysts and/or inactive, non-inflamed calcified cysts may be categorized as having unprovoked or remote seizures ${ }^{8}$. NC has an unpredictable clinical course, which makes it difficult to categorize all cases into the proposed classification of the ILAE Commission. A recent report from the ILAE defines comorbidity as the co-occurrence of two or more separate medical conditions in the same individual and includes $\mathrm{NC}$ as a comorbid condition associated with epilepsy ${ }^{4}$. Ultimately, in patients with $\mathrm{NC}$, it is critical to differentiate between provoked acute symptomatic seizures and recurrent unprovoked seizures (i.e., epilepsy).

The natural history of the cysticerci in the CNS is not entirely understood. Computed tomography (CT) and magnetic resonance imaging (MRI) have been useful in the study of the evolution of the cysticercus within the $\mathrm{CNS}^{9}$. Once the oncosphere has passed into the parenchyma, it grows and evolves through vesicular, colloidal, granular-nodular, and calcified phases. In the vesicular phase, the host tends to show immune tolerance and in most cases there is no surrounding parenchymal reaction; the larva lives inside a translucent liquid-filled cystic structure surrounded by a thin membrane, where it can remain viable from a few months to several years ${ }^{10}$. Figure depicts circumscribed, rounded, hypodense areas, varying in size and number, without enhancement by contrast media. With MRI, the vesicular larva appears with a CSF-like intensity signal on all sequences, with no surrounding high signal on T2-weighted images. Both MRI and CT may show a high intensity, 2-3 mm mural nodule, depicting the scolex, in the interior of some parenchymal vesicular cysts. As the cyst degenerates, it goes through a transitional or degenerative stage: the contrast enhanced CT scan shows an annular (colloidal phase) or nodular (granulo-nodular phase) enhancement surrounded by irregular perilesional edema ${ }^{9}$. In this stage, the fluid content gives slightly higher signal than CSF and is sometimes isodense with the parenchyma on MRI-T1 and/or proton density-weighted, and high signal on T2 images. The capsule shows higher signal than the adjacent brain with thick ring enhancement on T1 images, while on T2 images there is a low ring signal surrounded by high signal lesion, due mostly to edema. Although these pathological changes are responsible for clinical manifestations, such as seizure and headache, they may also be completely asymptomatic. Finally, when the cyst dies, it may disappear or become an inactive calcified nodule of homogenous high density on CT, or low intensity on proton-weighted $\mathrm{MRI}^{9,10}$.

A classification based on the viability and location of the parasite in the CNS has been proposed: active, when the parasite is alive; transitional or degenerative, if it is in the colloidal and granular-nodular phase; or inactive, if there is evidence of its death ${ }^{11}$. Each stage can be further classified based on cyst location (i.e., parenchymal or extraparenchymal). The viability criterion allows analysis of the natural history of the parasite and, according to the parasite's evolutionary stage, the production of pathophysiological changes in the CNS of the host. On the basis of this classification, it is possible to relate clinical manifestations to each stage of the proposed classification. For example, seizures are the main symptom in the presence of transitional cysts in the brain parenchyma due to the acute inflammatory reaction, whereas cranial nerve abnormalities and intracranial hypertension syndromes are more frequently observed in the pres- 
ence of subarachonoid and intraventricular cysts. It has been suggested that not all cysticercal granulomas are the result of degenerated vesicular cysts, but represent early or recently established metacestodes rapidly detected and destroyed by the host's immune system ${ }^{12}$. This is a hypothesis that should be confirmed by experimental research.

\section{EPIDEMIOLOGY OF NEUROCYSTICERCOSIS AND EPILEPSY}

T. solium infection is widely endemic in developing countries, both in highlands and tropical areas, throughout Latin America and among different regions of Asia and Africa ${ }^{1}$. It is important to note that not all developing countries are endemic for NC, for example, in predominately Islamic countries of Asia, Africa, and the Middle East where pork products are not consumed, $\mathrm{NC}$ is not endemic, and cases of NC may be imported or spread by incoming migration of $T$. solium carriers. NC is also considered an emerging infectious disease in some non-endemic countries due to migration and globalization ${ }^{2}$. Much of the data on the prevalence of $\mathrm{NC}$ in endemic countries has been compiled from prevalent cases from hospitals and clinics as well as other sources such as autopsied cadavers. However, such reports can be misleading and the lack of comprehensive and consistent reporting still limits attempts to compare the epidemiology of $\mathrm{NC}$ among countries ${ }^{13}$. An important issue is that the lack of validated diagnostic criteria for $\mathrm{NC}$ makes it difficult to estimate the prevalence of symptomatic $\mathrm{NC}^{14}$. Additionally, because NC can be completely asymptomatic, this makes it almost impossible to estimate what proportion of the population will actually have symptomatic disease. A systematic review by Ndimubanzi et al. attempted to determine the prevalence of $\mathrm{NC}$ based on studies using neuroimaging, but only found a limited number of studies to describe the prevalence of $\mathrm{NC}$ in select populations, concluding that the prevalence of $\mathrm{NC}$ worldwide remains unknown ${ }^{13}$.

Several studies have reported an association between epilepsy and NC based on positive serum antibodies against T. solium, which has distorted the perception of the epidemiology of epilepsy related to NC. Immunoserologic assays, such as enzyme-linked immunoelectrotransfer blot assay (EITB) or enzyme-linked immunosorbent assay (ELISA), detect antibodies against T. solium ${ }^{15}$. Epidemiological surveys using EITB report a seroprevalence of $8 \%$ to $12 \%$ for antibodies against T. solium in some regions of Latin America; however, most of these patients did not have symptomatic $\mathrm{NC}^{15,16}$. These assays are useful for identification of individuals who have had systemic contact with the parasite at some time, but their sole use in the diagnosis of $\mathrm{NC}$ is equivocal. Seropositivity does not necessarily implicate an active infection with $T$. solium, nor does it imply CNS involvement ${ }^{17}$. There are currently no reliable prospective cohort studies that provide information on the proportion of seropositive individuals that will develop seizures or other neurological symptoms. Furthermore, there are conflicting reports on an association between seropositivity and risk of seizures ${ }^{18,19}$. Although a higher proportion of patients with epilepsy have been shown to be EITB positive when compared with those without epilepsy, the proportion of seropositivity in epileptic patients is similar to that reported in the general population in these same areas ${ }^{16,18}$.

As with NC, the epidemiology of epilepsy depends heavily on the methodology and definitions used. In developing countries, the prevalence of epilepsy is about 8.5 cases per 1,000 persons, which is similar to developed countries ${ }^{20}$. However, the incidence of epilepsy in developing countries is much higher and is probably related to a higher frequency of risk factors, such as poor perinatal care, a higher frequency of head injury, and a high incidence of infectious diseases, such as $\mathrm{NC}^{20}$. Mortality is higher among persons with epilepsy in developing countries, which makes the prevalence of epilepsy similar among developing and developed countries ${ }^{20}$.

\section{SEIZURES AS THE MOST FREQUENT SYMPTOM OF NEUROCYSTICERCOSIS}

The heterogeneity of $\mathrm{NC}$ as demonstrated by its variable clinical presentations and evolution of cysts is well established ${ }^{15}$; however, the literature consistently support that seizures are the most common symptom of NC, occurring in $70 \%$ to $90 \%$ of symptomatic patients ${ }^{21}$. However, there is no uniformity in the reported distribution of types of seizure in patients with NC. Some authors report a higher proportion of focal seizures, while others conclude that generalized seizures are more frequent ${ }^{22,23}$. However, epidemiologic surveillance based on consistent reporting may be the only way to truly understand the distribution of types of seizures in patients with NC.

Seizures may occur at any evolutionary stage of the parasite, but NC-related seizures may be more common among children than adults ${ }^{24}$. It has been suggested that both age and gender influence the strength of the host's immune response ${ }^{24}$. In a recent study by Kelvin et al., the odds of having transitional cysts were found to be 1.5-fold higher for female patients than for male patients and the number of transitional cysts was 1.8-fold higher in females patients compared with male patients. This effect was not only statistically significant, but appeared to be constant over time. Some studies have also found familial aggregation of $\mathrm{NC}^{15,16}$, as well as regional differences in the clinical manifestations of $\mathrm{NC}$, which may indicate a role of host genetics. However, in a study carried out in a group of patients in 
Ecuador, there was no trend toward familial aggregation of seizures in patients with $\mathrm{NC}^{25}$.

Single enhancing lesion on CT (SECTL) or hyperintense lesion on MRI (Figure) is a common finding in patients with newly identified seizures in endemic countries ${ }^{26}$. These patients, mainly children and young adults, have some benign and transitory clinical manifestations, predominantly focal seizures or focal seizures with secondary generalization, and occasionally Todd's paresis or focal neurological deficits. These lesions have been attributed mainly to NC or to tuberculosis; however, similar lesions have been reported in other inflammatory pathologies such as pyogenic abscess, histoplasmosis, blastomycosis and sarcoidosis, post-infectious vasculitis, and to primary and metastatic brain tumors ${ }^{26}$. The natural history of these lesions usually takes one of two forms: it becomes isodense on CT, or isointense on MRI, and then either resolves entirely, or a punctuate calcification may be left as a residue ${ }^{26}$. The time until resolution of the lesion is quite variable ranging from a few weeks to more than a year. SECTL are benign and tend to resolve spontaneously, without antihelminthic drugs or surgery, since the parasite is already in the degenerative phase and will eventually disappear or become calcified ${ }^{1}$. Treatment for these patients should be limited to medications required to control the acute symptoms, such as antiepileptic drugs (AEDs) ${ }^{2}$.

\section{NEUROCYSTICERCOSIS AS AN ETIOLOGY OF EPILEPSY}

Prospective or case-control studies designed to establish associations and determinants of epilepsy related to $\mathrm{NC}$

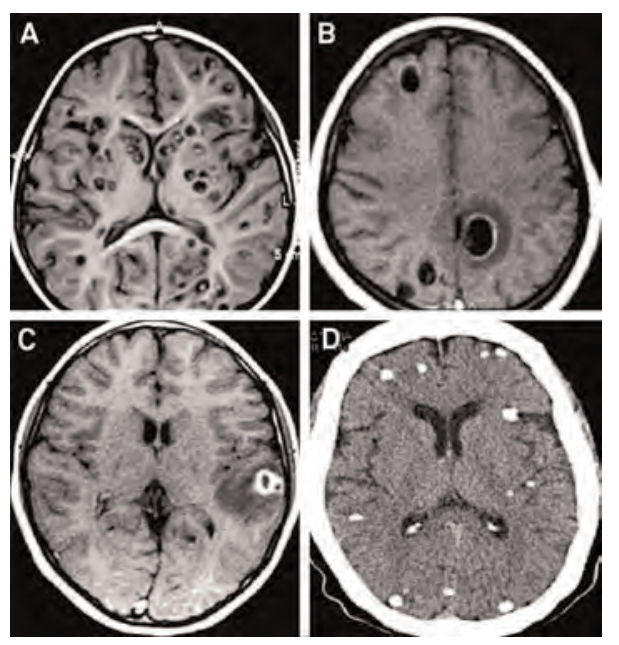

Figure. MRI and CT-scan of parenchymal cysticerci. MRI of vesicular cysticerci (A). MRI of colloidal cysticerci (B). Single hyperintense lesion on MRI (C). CT-scan of calcified cysticerci (D). are scarce. Comparing results of studies based on etiology is difficult because of the differences in definitions used (e.g., acute symptomatic seizure vs. epilepsy) and lack of validated diagnostic criteria for $\mathrm{NC}^{14}$. Most studies lack information on the latency between the first acute symptomatic seizure and the first unprovoked seizure, as well as age at onset of seizures and at diagnosis of NC. In studying etiology, it is preferable to use incident cases, not prevalent cases, because one cannot distinguish among the potential etiological factors that preceded the onset of epilepsy and thus, cause and effect become difficult to establish.

Although NC is a frequent antecedent in patients with epilepsy, it may not necessarily be the main cause of epilepsy, as has been previously suggested ${ }^{18,27,28}$. Studies of prevalent cases from what appear to be selected patients with seizures in neurology services reported $\mathrm{NC}$ as the main cause of epilepsy, accounting for $30 \%$ to $50 \%$ of patients ${ }^{27,28}$. However, in a study of patients with newly-diagnosed epilepsy seen at the five main hospitals in the three major cities of Ecuador, perinatal brain damage (9\%), NC (8.3\%), other CNS infections (4.2\%), stroke (4.8\%), and head trauma $(4.2 \%)$ were the most frequently reported as causes of epilepsy $^{29}$. In another study carried out in Ecuador, the authors concluded that $\mathrm{NC}$ is associated with one in three cases of epilepsy; however, only $8 \%(3 / 24)$ of persons with epilepsy had a diagnosis of "definitive" $\mathrm{NC}^{28}$. Another community survey carried out in Peru showed that seroprevalence, using EITB, was positive in $24.2 \%$ (200/825) of individuals and $38.5 \%(15 / 39)$ of individuals with seizures had lesions compatible with $\mathrm{NC}$ on $\mathrm{CT}$ scan $^{18}$. The authors concluded that these CT abnormalities consistent with NC were more frequent in individuals with seizures and in those with positive EITB. Most of the patients who were diagnosed with NC in this study had only calcified lesions with half of patients having just one calcification. Nevertheless, the presence of calcified lesions does not necessarily mean that seizures were provoked by $\mathrm{NC}$, particularly in studies of prevalent cases. Epidemiologic studies have been carried out in SubSaharan Africa and India; however, as with studies from Latin America, their results are difficult to draw definitive conclusions from ${ }^{30,31}$.

These studies demonstrate the need to differentiate between acute seizures and recurrent unprovoked seizures (i.e., epilepsy) when implicating NC in the etiology of seizures or epilepsy. Although acute symptomatic seizures are a common symptom of NC, they may not necessarily develop into epilepsy. Similarly, it is critical to determine $\mathrm{NC}$ as an etiology of epilepsy using incident cases, as estimation from prevalent cases may lead to overestimation of NCrelated epilepsy. Multiple studies have reported an association between epilepsy and NC based on positive serum antibodies to T. solium $^{32,33}$. Unfortunately, the presence of 
antibodies may only indicate previous exposure to the parasite, but not necessarily infection of the CNS. Because of the high prevalence of NC and epilepsy, a causal, as well as incidental relationship between the two conditions might exist $^{34,35}$. NC is also an uncommon cause of intractable epilepsy, even in endemic regions and may only represent a coexistent pathology as demonstrated by a cross-sectional study investigating the etiology of intractable epilepsy in 512 patients in Brazil ${ }^{36}$.

\section{EPILEPTOGENESIS OF NEUROCYSTICERCOSIS LESIONS}

There are clinical inconsistencies in the causal link between epilepsy and NC lesions. Parasite location may be remote from the apparent epileptogenic region and there is no apparent correlation between the burden of lesions and the severity of the epilepsy ${ }^{8}$. For example, patients with severe refractory seizures may have only one calcified lesion and patients with multiple cysts or calcifications may have no seizures. Electroencephalography (EEG) has been found to be abnormal in $30 \%$ to $50 \%$ of patients with seizures due to NC and thus, EEG findings may have poor correlation with symptoms and $\mathrm{CT}$ lesions in patients with $\mathrm{NC}^{37,38}$. Discrepancies between clinical localization based on seizure semiology and location of the lesion on neuroimaging is not uncommon in patients with NC. An incidental relationship between epilepsy and NC, in some cases, might explain these apparent discrepancies.

The mechanism by which the calcified lesions cause seizures or epilepsy is not known ${ }^{1,39,40}$, but it has been attributed to residual perilesional gliosis that results in chronic epileptogenic foci ${ }^{34}$. Calcified lesions are frequently encountered in CT scans of asymptomatic individuals, and studies from Latin American countries report that the majority are incidental lesions, which questions the true epileptogenicity of calcified lesions ${ }^{15,34,41}$. However, based on epidemiological studies, patients with epilepsy are more likely to have calcified lesions present compared with controls without epilepsy $^{42}$. In addition to perilesional gliosis, perilesional edema associated with calcified lesions has also been implicated by episodic appearance of edema surrounding the lesion after seizures ${ }^{43}$. Nash et al. propose that cysticercal antigens from calcified lesions can lead to inflammation, perilesional edema, and seizures ${ }^{43}$. However, it is not clear whether this edema is the cause or the consequence of seiz$u_{r}{ }^{34}$. Transient cortical edema after seizures, a rare neuroimaging finding, was described more than 20 years ago, but its mechanism has not been entirely clarified ${ }^{44}$. The empirical observation of neurologists from endemic countries is that perilesional edema around calcified lesions in patients with epilepsy is extremely rare and, when it happens, perilesional edema disappears in a few days without any additional treatment, except for AEDs.

Degree of blood brain barrier permeability may also play a role in epileptogenesis of calcified lesions. A study designed to evaluate epileptogenesis of calcified lesions using dynamic contrast-enhanced MRI, reported that median values of the rate transfer constant and leakage volume were higher in symptomatic patients than in asymptomatic patients, indicating a higher degree of blood brain barrier permeability in symptomatic individuals ${ }^{45}$. This study also showed increased MMP-9 (R279Q) polymorphisms in subjects with seizures compared with asymptomatic patients and controls ${ }^{45}$. Therefore, the epileptogenicity of calcified lesions could be related to degree of inflammation, which also may be partly determined by genetic factors. This study was carried out in a small sample of patients and necessitates replication.

The coexistence of hippocampal atrophy and extrahippocampal pathological abnormalities, such as cortical dysgenesis and gliosis, referred to as "dual pathology", has been reported in $5 \%$ to $30 \%$ of patients with medically refractory focal seizures $^{46}$. Dual pathology implies that both lesions somehow interact with each other and contribute to epileptogenesis through mechanisms which remain poorly understood. Some authors have also attributed hippocampal sclerosis to $\mathrm{NC}^{47,48}$. However, in one study, patients with calcified lesions and mesial temporal lobe epilepsy (i.e., hippocampal sclerosis) became seizure free after anteromesial temporal lobectomy, without resection of the calcified lesions ${ }^{34}$, suggesting the two phenomena are independent. The presence of calcified lesions does not influence the clinical and pathologic profile of patients with hippocampal atrophy. An irritative zone in the temporal lobe is more relevant in determining the severity, symptomatology and frequency of seizures rather than the number and location of calcifications ${ }^{34,49}$. The possibility of dual pathology related to NC needs further clarification in prospective cohort studies.

A recent study by Rathore et al. concluded that calcified NC lesions are potential cause for AED-resistant and surgically remediable epilepsy, as well as dual pathology ${ }^{48}$. However, this report has methodological issues, related to sample selection and surgical candidacy, which limit the generalizability of these findings. In general, NC has a favorable prognosis in terms of seizure recurrence, therefore, calcified lesions should not be surgically removed, with the exception of such lesions located in the hippocampus, since we do not know if the benefits of surgery outweigh the risks. The current evidence regarding the epileptogenecity of NC and specifically the role of calcified lesions in AED-resistant epilepsy is scarce and based on anecdotal cases. A prospective cohort study is needed to assess the association of different evolutive phases of the parasite and the development of seizures, as well as the evolution of NC cysts after medical (and/or surgical) treatment. 


\section{ANTIHELMINTHIC TREATMENT AND PROGNOSIS OF SEIZURES IN NEUROCYSTICERCOSIS}

Pharmacotherapy for NC with antihelminthic drugs such as praziquantel and albendazole has been available for at least 25 years. Despite the existence of randomized controlled trials comparing antihelminthic drugs and placebo, these studies remain limited by the fact that the doses and duration of drugs tested are empiric, and not optimized through dose finding studies specific to $\mathrm{NC}^{50}$. Furthermore, a benefit in reduction of seizure risk, a clinically important outcome in NC, remains to be established. A randomized controlled trial carried out to analyze whether treatment with albendazole results in improved seizure control in patients with $\mathrm{NC}$ reported a significant reduction of the number of "seizures with generalization" (which included generalized seizures, partial seizures with secondary generalization, and complex partial seizures) compared with placebo, but did not find any significant difference between albendazole and placebo when analyzing all seizures $(\text { Table })^{51}$.

In addition to reduction of seizure risk, complete disappearance of active cysts is another important endpoint for evaluating treatment of NC. In an early meta-analysis of trials evaluating praziquantel or albendazole, only 11 studies were included, of which 5 were considered to be of good quality $^{52}$. Six of the eleven studies included patients with active cysts and 5 included patients with degenerative or colloidal cysts. The authors concluded that the studies included provided limited evidence of a modest effect of antihelminthic treatment for $\mathrm{NC}$ considering that the effects of treatment on neuroimaging endpoints were relatively small $^{52}$. A more recent Cochrane review of 21 randomized controlled trials of antihelminthic treatment concluded that in adults with viable cysts, the use of albendazole is associated with a decrease in the number of cysts but not in the recurrence of seizures ${ }^{53}$.

One of the sources of confusion surrounding efficacy of antihelminthic treatment is defining successful treatment. Reporting "reduction of the number of lesions" as a valid endpoint to measure effectiveness can be misleading. In the hypothetical event that a patient with 10 viable cysts has been administered antihelminthic treatment and 2 viable cysts remain, is an $80 \%$ reduction in number of viable cysts a successful treatment? If two cysts remain, they may provoke seizures or other neurologic symptoms of NC, and thus, treatment was a failure. The most appropriate endpoint for evaluating the effectiveness of antihelminthic treatment on cysts should be considered the complete disappearance of active cysts. Strictly speaking, it is not possible to talk about "cure" when describing reduction of cysts because most cysts that degenerate leave a permanent calcification, which has the potential to cause future seizures.

If we compare randomized placebo-controlled trials, in which the endpoint to evaluate effectiveness of antihelminthic treatment was disappearance of cysts, about onethird of patients had disappearance of cysts (Table $)^{51,53,54,55}$. If we look at these trials individually, antihelminthic treatment in patients with seizures due to $\mathrm{NC}$ is not associated with improvement in the remission of seizures (Table $)^{51,53,54,55}$. Despite the lack of statistically significant effect of antihelminthic drugs on seizure recurrence, acute symptomatic seizures related to NC generally have good prognosis in terms of remission ${ }^{56,57}$. The risk of seizure recurrence after a first seizure due to $\mathrm{NC}$ is between $17 \%$ and $56 \%$, depending on the viability of the parasite, with greater risk with transitional cysts and diminished risk with viable or

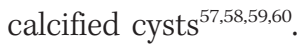

During antihelminthic treatment of $\mathrm{NC}$, AEDs are routinely coadministered to prevent seizures; however, AEDs, such as phenytoin and carbamazepine, may significantly reduce plasma concentrations of albendazole and praziquantel $^{50}$. The clinical relevance of this drug interaction remains to be determined (i.e., if lower levels of albendazole or praziquantel contribute to treatment failure $)^{50}$. Regarding the duration that AEDs should be continued following acute seizures related to $\mathrm{NC}$, some clinicians routinely continue AEDs for 1 year, but both shorter and longer intervals have been recommended ${ }^{1}$. One can assume that the risk of seizures is substantial as long as there is an active, ongoing process as characterized by persistence of edema around the degenerating lesion. Because of this, CT is a useful tool for

Table. Effects of albendazole on resolution of viable parenchymal cysts and seizures recurrence.

\begin{tabular}{lccc}
\hline Author/Year & Treatment groups & $\begin{array}{c}\text { Patients with disappearance of viable cysts* } \\
\text { No. patients (\%) }\end{array}$ & $\begin{array}{c}\text { Seizure recurrence** } \\
\text { No. patients (\%) }\end{array}$ \\
\hline Garcia et al. 200451 & Albendazole & $21 / 57(37 \%)$ & $32 / 57(56 \%)$ \\
& Placebo & $8 / 59(14 \%)$ & $32 / 59(54 \%)$ \\
Das et al. 200755 & Albendazole & $10 / 148(7 \%)$ & $32 / 148(22 \%)$ \\
& Placebo & $12 / 150(8 \%)$ & $15 / 150(10 \%)$ \\
Carpio et al. 200854 & Albendazole & $19 / 39(49 \%)$ & $23 / 50(47 \%)$ \\
& Placebo & $8 / 27(23 \%)$ & $28 / 56(53 \%)$ \\
\hline
\end{tabular}

*Imaging at 6 months after finishing treatment. **Seizures recurrence at one year follow-up for Das et al. and Carpio et al.; seizure recurrence at 30 months' follow-up for Garcia et al. 
these treatment decisions. It is appropriate to monitor cyst activity with CT scanning and to continue AEDs until resolution of the acute lesion, after which AEDs may be discontinued $^{1}$. Seizures occurring in individuals after resolution of edema or calcification of the degenerating cyst should be considered unprovoked and, in this situation, long-term AED use is warranted.

\section{CONCLUSIONS}

$\mathrm{NC}$ is a highly heterogeneous disease and important cause of seizures and epilepsy. Despite this, cause and effect need to be cautiously interpreted from studies, as both NC and epilepsy can be related or may be independent processes. There are many unanswered questions with regard to the epileptogenicity of NC. Determining the underlying mechanism of epilepsy related to calcified NC lesions is critical. Furthermore, the interpretation of risk of seizures and epilepsy in patients with NC is difficult. These difficulties are increased in those patients who have mixed forms, including active, degenerative, and calcified lesions. Further studies should be performed in order to estimate recurrence risk in those patients with seizures due to calcifications alone in comparison with patients with acute seizures due to degenerative cysts. Antihelminthic treatment of $\mathrm{NC}$ appears to only be effective in eradicating active cysts in about one-third of patients and thus, more efficacious treatment strategies are needed. Ultimately, health authorities should focus on prevention and eradication of cysticercosis to decrease the number of individuals with seizures and epilepsy in endemic countries.

\section{References}

1. Carpio A. Neurocysticercosis: an update. Lancet Infect Dis 2002;2:751-762.

2. Pal DK, Carpio A, Sander JW. Neurocysticercosis and epilepsy in developing countries. J Neurol Neurosurg Psychiatry 2000;68:137-143.

3. Fabiani S, Bruschi F. Neurocysticercosis in Europe: Still a public health concern not only for imported cases. Acta Trop 2013;128:18-26.

4. Thurman DJ, Beghi E, Begley CE, et al. Standards for epidemiologic studies and surveillance of epilepsy. Epilepsia 2011;52(Suppl 7):S2-S26.

5. Berg AT, Scheffer IE. New concepts in classification of the epilepsies: entering the $21^{\text {st }}$ century. Epilepsia 2011;52:1058-1062.

6. Fisher RS, van Emde Boas W, Blume W, et al. Epileptic seizures and epilepsy: definitions proposed by the International League Against Epilepsy (ILAE) and the International Bureau for Epilepsy (IBE). Epilepsia 2005;46:470-472

7. Guidelines for epidemiologic studies on epilepsy. Commission on Epidemiology and Prognosis, International League Against Epilepsy. Epilepsia 1993;34:592-596.

8. Carpio A, Escobar A, Hauser WA. Cysticercosis and epilepsy: a critical review. Epilepsia 1998;39:1025-1040.

9. Carpio A, Placenua M. Definition of contemporary surgical management in cisternal and parenchymatous cysticercosis cerebri. Neurosurgery 1992;30:968-969.

10. Santos GT, Leite CC, Machado LR, McKinney AM, Lucato LT. Reduced diffusion in neurocysticercosis: circumstances of appearance and possible natural history implications. AJNR Am J Neuroradiol 2013;34:310-316

11. Carpio A, Placencia M, Santillan F, Escobar A. A proposal for classification of neurocysticercosis. Can J Neurol Sci 1994;21:43-47.

12. Garcia HH, Gonzalez AE, Rodriguez $S$ et al. Neurocysticercosis: unraveling the nature of the single cysticercal granuloma. Neurology 2010;75:654-658

13. Ndimubanzi PC, Carabin H, Budke CM, et al. A systematic review of the frequency of neurocyticercosis with a focus on people with epilepsy. PLoS Negl Trop Dis 2010;4:e870.

14. Machado LR. The diagnosis of neurocysticercosis: a closed question? Arq Neuropsiquiatr 2010;68:1-2

15. Fleury A, Escobar A, Fragoso G, Sciutto E, Larralde C. Clinical heterogeneity of human neurocysticercosis results from complex interactions among parasite, host and environmental factors. Trans R Soc Trop Med Hyg 2010;104:243-250.
16. Goodman KA, Ballagh SA, Carpio A. Case-control study of seropositivity for cysticercosis in Cuenca, Ecuador. Am J Trop Med Hyg 1999;60:70-74.

17. Carpio A, Fleury A, Hauser WA. Neurocysticercosis: Five new things. Neurol Clin Pract 2013;3:118-125

18. Montano SM, Villaran MV, Ylquimiche L, et al. Neurocysticercosis: association between seizures, serology, and brain CT in rural Peru. Neurology 2005;65:229-233.

19. Garcia-Noval J, Allan JC, Fletes C, et al. Epidemiology of Taenia solium taeniasis and cysticercosis in two rural Guatemalan communities. Am J Trop Med Hyg 1996;55:282-289.

20. Carpio A, Hauser WA. Epilepsy in the developing world. Curr Neurol Neurosci Rep 2009;9:319-326.

21. Carabin $\mathrm{H}$, Ndimubanzi PC, Budke CM, et al. Clinical manifestations associated with neurocysticercosis: a systematic review. PLoS Negl Trop Dis 2011;5:e1152.

22. Murthy JM, Reddy VS. Clinical characteristics, seizure spread patterns and prognosis of seizures associated with a single small cerebral calcific CT lesion. Seizure 1998;7:153-157.

23. Martinez HR, Rangel-Guerra R, Arredondo-Estrada JH, Marfil A, Onofre J. Medical and surgical treatment in neurocysticercosis a magnetic resonance study of 161 cases. J Neurol Sci 1995;130:25-34.

24. Kelvin EA, Carpio A, Bagiella E, et al. The association of host age and gender with inflammation around neurocysticercosis cysts. Ann Trop Med Parasitol 2009;103:487-499.

25. Kelvin EA, Carpio A, Hesdorffer DC, et al. Investigation of familial aggregation of seizures in neurocysticercosis patients. Epilepsy Res 2009;84:67-71.

26. Singh G, Rajshekhar V, Murthy JM, et al. A diagnostic and therapeutic scheme for a solitary cysticercus granuloma. Neurology 2010;75:2236-2245

27. Medina MT, Rosas E, Rubio-Donnadieu F, Sotelo J. Neurocysticercosis as the main cause of late-onset epilepsy in Mexico. Arch Intern Med 1990;150:325-327.

28. Del Brutto $\mathrm{OH}$, Santibanez R, Idrovo L, et al. Epilepsy and neurocysticercosis in Atahualpa: a door-to-door survey in rural coastal Ecuador. Epilepsia 2005;46:583-587.

29. Carpio A, Hauser WA. Remote symptomatic epilepsy and epileptic syndromes: etiological spectrum. In: Murthy JMK SN (Ed). Epilepsy in the Tropics. Georgetown, USA: Landes Bioscience; 2006:72-84. 
30. Winkler AS, Blocher J, Auer H, Gotwald T, Matuja W, Schmutzhard E. Anticysticercal and antitoxocaral antibodies in people with epilepsy in rural Tanzania. Trans R Soc Trop Med Hyg 2008;102:1032-1038.

31. Singh G, Singh P, Singh I, Rani A, Kaushal S, Avasthi G. Epidemiologic classification of seizures associated with neurocysticercosis: observations from a sample of seizure disorders in neurologic care in India. Acta Neurol Scand 2006;113:233-240.

32. Prasad KN, Prasad A, Gupta RK, et al. Neurocysticercosis in patients with active epilepsy from the pig farming community of Lucknow district, north India. Trans R Soc Trop Med Hyg 2009;103:144-150.

33. Blocher J, Schmutzhard E, Wilkins PP, et al. A cross-sectional study of people with epilepsy and neurocysticercosis in Tanzania: clinical characteristics and diagnostic approaches. PLoS Negl Trop Dis 2011;5:e1185.

34. Leite JP, Terra-Bustamante VC, Fernandes RM, et al. Calcified neurocysticercotic lesions and postsurgery seizure control in temporal lobe epilepsy. Neurology 2000;55:1485-1491.

35. Terra-Bustamante VC, Coimbra ER, Rezek KO, et al. Cognitive performance of patients with mesial temporal lobe epilepsy and incidental calcified neurocysticercosis. I Neurol Neurosurg Psychiatry 2005;76:1080-1083.

36. Velasco TR, Zanello PA, Dalmagro CL, et al. Calcified cysticercotic lesions and intractable epilepsy: a cross sectional study of 512 patients. J Neurol Neurosurg Psychiatry 2006;77:485-488.

37. Chayasirisobhon S, Menoni R, Chayasirisobhon W, Locke GE. Correlation of electroencephalography and the active and inactive forms of neurocysticercosis. Clin Electroencephalogr 1999;30:9-11.

38. Cukiert A, Puglia P, Scapolan HB, Vilela MM, Marino Junior R. Congruence of the topography of intracranial calcifications and epileptic foci. Arq Neuropsiquiatr 1994;52:289-294.

39. Antoniuk SA, Bruck I, Dos Santos LH, et al. Seizures associated with calcifications and edema in neurocysticercosis. Pediatr Neurol 2001;25:309-311.

40. Rathore C, Thomas B, Kesavadas C, Radhakrishnan K. Calcified neurocysticercosis lesions and hippocampal sclerosis: potential dual pathology? Epilepsia 2012;53:60-62.

41. Kowacs PA, Rogacheski E, Muzzio J, Werneck LC. The role of the irritative zone and of the number and distribution of calcifications in the severity of epilepsy associated with intracranial calcifications. Arq Neuropsiquiatr 2006;64:905-911.

42. Garcia-Noval J, Moreno E, de Mata F, et al. An epidemiological study of epilepsy and epileptic seizures in two rural Guatemalan communities. Ann Trop Med Parasitol 2001;95:167-175.

43. Nash TE, Pretell EJ, Lescano AG, et al. Perilesional brain oedema and seizure activity in patients with calcified neurocysticercosis: a prospective cohort and nested case-control study. Lancet Neurol 2008;7:1099-1105.

44. Bansal BC, Dua A, Gupta R, Gupta MS. Appearing and disappearing CT scan abnormalities in epilepsy in India - an enigma. J Neurol Neurosurg Psychiatry 1989;52:1185-1187.
45. Gupta RK, Awasthi R, Rathore RK, et al. Understanding epileptogenesis in calcified neurocysticercosis with perfusion MRI. Neurology 2012;78:618-625.

46. Cendes F, Cook MJ, Watson C, et al. Frequency and characteristics of dual pathology in patients with lesional epilepsy. Neurology 1995; $45: 2058-2064$

47. Singla M, Singh $P$, Kaushal S, Bansal R, Singh G. Hippocampa sclerosis in association with neurocysticercosis. Epileptic Disord 2007;9:292-299

48. Rathore C, Thomas B, Kesavadas C, Abraham M, Radhakrishnan K. Calcified neurocysticercosis lesions and antiepileptic drug resistant epilepsy: a surgically remediable syndrome? Epilepsia 2013;54:1815-1822

49. Singh G, Burneo JG, Sander JW. From seizures to epilepsy and its substrates: neurocysticercosis. Epilepsia 2013;54:783-792.

50. Romo ML, Carpio A, Kelvin EA. Routine drug and food interactions during antihelminthic treatment of neurocysticercosis: a reason for the variable efficacy of albendazole and praziquantel? J Clin Pharmacol 2014;54:361-367.

51. Garcia HH, Pretell EJ, Gilman RH, et al. A trial of antiparasitic treatment to reduce the rate of seizures due to cerebral cysticercosis. N Engl J Med 2004;350:249-258.

52. Del Brutto OH, Roos KL, Coffey CS, Garcia HH. Meta-analysis: cysticidal drugs for neurocysticercosis: albendazole and praziquantel. Ann Intern Med 2006;145:43-51.

53. Abba K, Ramaratnam S, Ranganathan LN. Anthelmintics for people with neurocysticercosis. Cochrane Database Syst Rev 2010:CD000215.

54. Carpio A, Kelvin EA, Bagiella E, et al. Effects of albendazole treatment on neurocysticercosis: a randomised controlled trial. J Neurol Neurosurg Psychiatry 2008;79:1050-1055.

55. Das K, Mondal GP, Banerjee M, Mukherjee BB, Singh OP. Role of antiparasitic therapy for seizures and resolution of lesions in neurocysticercosis patients: an 8 year randomised study. J Clin Neurosci 2007;14:1172-1177.

56. Singhi P, Ray M, Singhi S, Khandelwal N. Clinical spectrum of 500 children with neurocysticercosis and response to albendazole therapy. J Child Neurol 2000;15:207-213.

57. Carpio A, Hauser WA. Prognosis for seizure recurrence in patients with newly diagnosed neurocysticercosis. Neurology 2002;59:1730-1734.

58. Sharma P, Garg RK, Verma R, Singh MK, Shukla R. Risk of seizure recurrence in patients of new-onset partial seizure having a solitary cysticercus granuloma of brain or normal neuroimaging. J Neurol Sci 2011;301:21-26.

59. Thussu A, Chattopadhyay A, Sawhney IM, Khandelwal N. Albendazole therapy for single small enhancing CT lesions (SSECTL) in the brain in epilepsy. J Neurol Neurosurg Psychiatry 2008;79:272-275

60. de Souza A, Thennarasu K, Yeshraj G, Kovoor JM, Nalini A. Randomized controlled trial of albendazole in new onset epilepsy and MRI confirmed solitary cerebral cysticercal lesion: effect on long-term seizure outcome. J Neurol Sci 2009;276:108-114. 